\title{
MODEL OF SLOWLY EVOLVING FLARE
}

\author{
F. Chiuderi Drago ${ }^{1}$, M.Landini $^{2}$ and B.C.Monsignori Fossi ${ }^{3}$ \\ I Institute of Astronomy, University of Florence, Italy \\ 2 Department of Physics, University of Naples, Italy \\ ${ }^{3}$ Arcetri Astrophysical Observatory, Florence, Italy
}

\begin{abstract}
A gradual rise and fall flare with a duration of about one hour was observed on June 10 , 1950 in the radio (Toyokawa and VLA), optical (Bing Bear) and XUV (SMM1 satellite) ranges of wavelengths. The flare developed as a large loop connecting two regions of opposite polarity in a preexisisting acrive region. A model of the differential emission measure of the loop obserwed at three different stage of the flare is deduced from the analysis of the XUV images in $C \mathrm{~N}^{\prime}(15+9 \mathrm{~A}), \mathrm{OVIII}$ $(18.97 \mathrm{~A}), \mathrm{Ne} I X(13.45 \mathrm{~A}), \mathrm{Mg} X I(9.17 \mathrm{~A})$ and Si XIII $(6.65 \mathrm{~A})$ emission lines. The differential emission measure as a function of temperature is controlled by the conductive flux via the temperature gradient; the evaluation of the divergence of the conductive flux is used in the energy balance to have information on the power deposition function.
\end{abstract}

\section{OBSERVATIONS}

Preliminary results of the analysis of a solar flare, which took place on Junc 10/11, 1980 and was observed in all ranges of wavelengths from X-ray to radio, are presented. In this analysis only UVSP: CIV and XRP: FCS Ch. 1 to 4 rasters are taken into account. The peculiar characteristic of the fare is the estremely slow rate of intensity increase and fall and its long duration (about one hour).

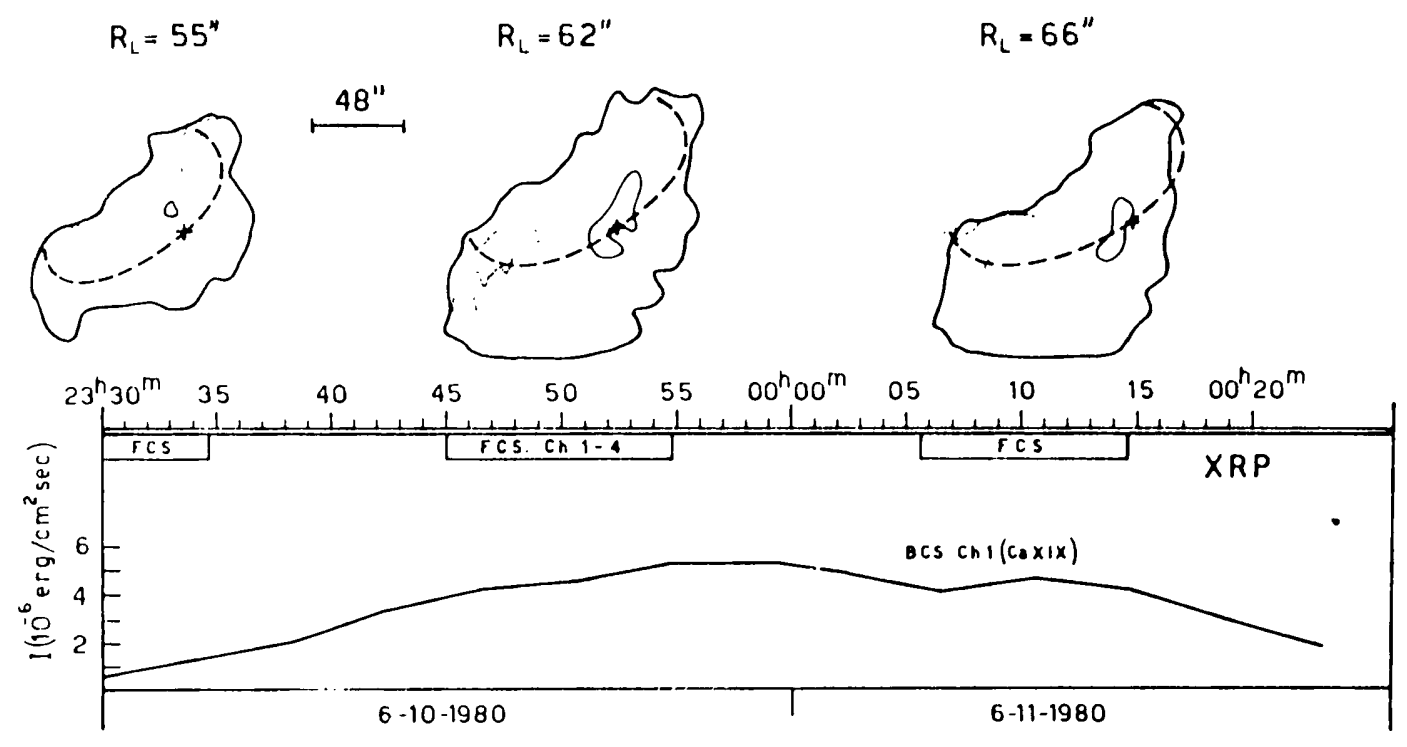

fig.1

In fig. 1 the time behaviour of the whole flare intensity, as observed by XRP:BCS (lower panel), is presented, the windows indicate the time intervals in which XRP:FCS rasters and UVSP CIV 
dopplegrams are available. In the upper pannel the morphology of the flare resulting from UVSP and XRP:FCS contour levels, recorded in three different time intervals, is shown: XRP contour levels correspond to the level inside which the flux has been integrated in all spectral lines (thick line) and the level corrisponding to $0.8 \mathrm{I}_{\text {inax }}$ in channcl 4 [SiXIII] (thin linc). UVSP (dotted line) corrispond to contour level $0.1 \mathrm{I}_{\mathrm{max}}$. The dashed line is the projection on the disk of a semicircular loop, of radius $R_{L}$ lying in the local vertical plane, and the cross is the projection of its maximum. Notice the coincidence between the cross and the maximum of Si XIII.

\section{TIEE LOOP MODEL}

The theoretical luminosity $D(\lambda)\left(\mathrm{crg} \mathrm{s}^{-1}\right)$ in an optically thin line is given by:

$$
\Phi(\lambda)=\int_{T_{1}}^{T_{c}} f(\lambda, T) n^{2} d V
$$

where $f(\lambda, T)$ is the power emitted per unit emission measure in the line, $\mathrm{n}$ is the electron density in the volume $\mathrm{dV}, \mathrm{T}_{\mathrm{c}}$ and $\mathrm{T}_{1}$ are the maximum and minimum temperature of the loop. For $\mathrm{T}<\mathrm{T}_{1}=2.10^{4} \mathrm{o}_{\mathrm{K}}$ the assumption of optically thin line is no longer valid. The power emitted per unit emission measure $\mathrm{r}(\lambda, T)$ has been evaluated by means of a computer code developped by Landini and Monsignori Fossi (1984) for low density and high temperature plasmas.

Loop models for the solar active regions have been extensively investigated in the last 15 years (Rosner et al.1978, Landini et al.1985 and references therein). Most of these studies consist in the cvaluation of the differential emission measure (d.c.m. $=n^{2} \mathrm{dV} / \mathrm{dT}$ ) as a function of temperature from which the theorelical line intensity can be computed and compared with observations. The differential cmission measure (d.c.m.) dipends on the temperature gradient which is defined by the conductive flux. For instance in a stationary coronal loop with costant pressure $p_{O}$ and costant cross section $S$, the energy ballance equation gives the following lorm for the conductive flux :

$$
\mathrm{F}=-\mathrm{A} \mathrm{T}^{2.5} \mathrm{dT} / \mathrm{dl}=\mathrm{F}_{\mathrm{o}}\left(\mathrm{T} / \mathrm{T}_{\mathrm{c}}\right)^{\beta}\left[\left(\mathrm{l}-\left(\mathrm{T} / \mathrm{T}_{\mathrm{C}}\right)^{\left.\delta_{\mathrm{S}}\right] / 2}\right.\right.
$$

In eal. 21 is the cordinate along the loop, $\beta$ depends on the radiative losses approximation and $\delta$ on the specific power law approximation used for the energy supply (for istance radiative losses proportional to $\mathrm{n}^{2} \mathrm{~T}^{-0.5}$ give $\beta=0.5$ and power supply indipendent on the temperature gives $\delta=2.5$ ).

Deriving, in the above assumption, the d.c.m. from eq. 2 and putting it in eq.1, high temperature line intensities are very well reproduced, while low temperature one $\left(\mathrm{T}<3.10^{5} \mathrm{~K}\right.$ ) are strongly understimated.This fact have suggested to Monsignori Fossi and Landini (1988) 10 multiply the rigth hand side of eq..2 by an exponential term: $\exp \left(T / T_{0}\right)$ where $T_{0}$ is a free parameter $\left(T_{1}<T_{0}<T_{C}\right)$. With this form of $F$ and the assumption $p_{0}=$ const., the d.e.m. becomes:

$$
\text { d.c..m. } \left.=n^{2} \mathrm{aV} / \mathrm{dT}=\left(\mathrm{p}_{0}{ }^{2} / 4 \mathrm{k}^{2}\right)\left(10^{-6} / \mathrm{F}_{\mathrm{O}}\right) \mathrm{T}^{0.5}\left(\mathrm{~T} / \mathrm{T}_{\mathrm{c}}\right)^{-\beta}(\exp )\left(\mathrm{T}_{\mathrm{O}} / \mathrm{T}\right)\left[1-\left(\mathrm{T} / \mathrm{T}_{\mathrm{c}}\right)^{\delta}\right]^{-1 / 2}\right]
$$

Assuming $\log T_{0}=5.2$, the theoretical mean intensity $\left(\mathrm{crg} \mathrm{cm}^{-2} \mathrm{~s}^{-1} \mathrm{st}^{-1}\right)$ in each line is evaluated and the frec parmeters $\beta, \delta, T_{c}$ and the normalizalion factor $S p_{0}{ }^{2} / F_{0}$ are deduced from a $\chi^{2}$ lit with the observations.

Table 1

$\begin{array}{lclcll}\text { Time } & \chi^{2} & \log \mathrm{T}_{\mathrm{c}} & \delta & \beta & S \mathrm{p}_{\mathrm{o}}^{2} / \mathrm{F}_{\mathrm{o}} \\ 23^{\mathrm{h}} 26^{\mathrm{m}} & .6 & 6.63 \pm .02 & 0.5 \pm .1 & 0.1 \pm .15 & 9.510^{12} \\ 23^{\mathrm{h}} .15^{\mathrm{m}} & 1.5 & 6.72 \pm .03 & 0.5 \pm .1 & 0.2 \pm .2 & 1.510^{13} \\ 00^{\mathrm{h}} 06^{\mathrm{m}} & 1 . & 6.74 \pm .02 & 0.5 \pm .1 & 0.15 \pm .15 & 2.410^{13}\end{array}$


The procedure has been applied to the three sets of data show'n in fig.1 (top) to have an insight of the time variation of the loop structure during the event. The results of the fit are given in the table 1. Uncertainty of the normalization factor $\mathrm{Sp}_{\mathrm{O}} 2 / \mathrm{F}_{\mathrm{O}}$ is about $\pm 20 \%$.

By integration of the d.c.m. function the temperature profile may be obtaincd: fig 2 shows the temperature profile for the time $00^{\text {h }} 06^{\mathrm{m}}$ U.T.: vertical bars give the temperature derived from the averaged line intensity in each section of $10^{\prime \prime}$ thickness, perpendicular to the loop length and assumed isothermal.

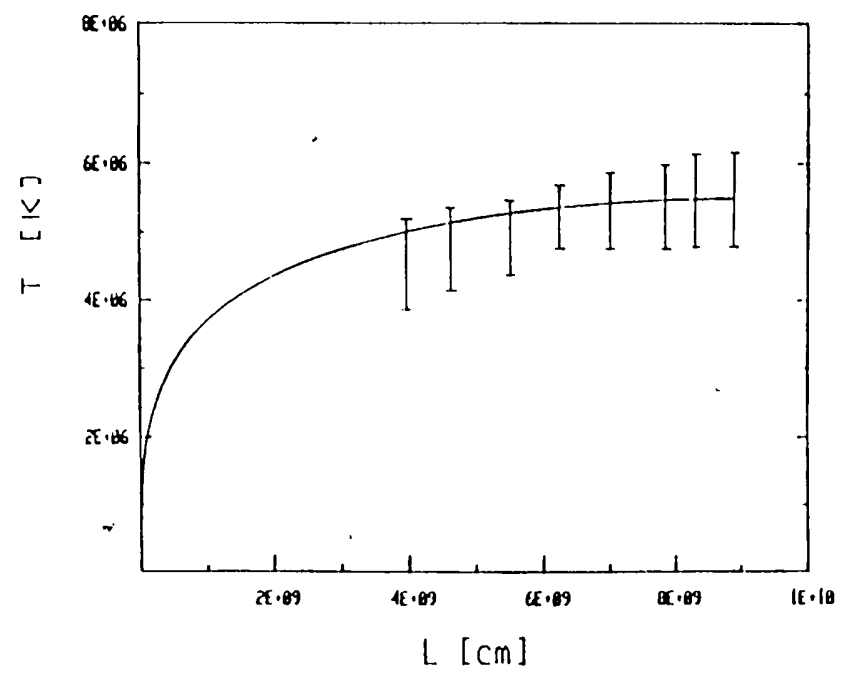

lig. 2

Since the length $l$ and the section $S$ can been measured on the images, $F_{0}$ and the pressure $p_{0}$ may be evaluated. Uncertaitics of $F_{0}$ and $p_{0}$ amount to $\pm 15 \%$. Table 2 shows, for each lime interval, the semilength and section of the loop and the pressure

Tablc 2

$\begin{array}{lllc}\text { Time } & 1 / 2(\mathrm{~cm}) & S\left(\mathrm{~cm}^{2}\right) & p_{0}\left(\text { dyn } / \mathrm{cm}^{2}\right) \\ 23^{\mathrm{h}} 26^{\mathrm{m}} & 7.910^{9} & 2.810^{18} & 10 . \\ 23^{\mathrm{h}} 45^{\mathrm{m}} & 8.610^{9} & 4.410^{18} & 15 . \\ 00^{\mathrm{h}} 06^{\mathrm{m}} & 8.910^{9} & 4.410^{18} & 18 .\end{array}$

Table 1 and 2 show that the pressure increases by about a fuctor of two, while the maximum temperature increases less than $30 \%$.

The small variation presented by the parameters entering in the model justilies the use of a stationary model to study this event. For each time interval the conductive flux and its divergence may be computed from the temperature profile; the temperature profile also allows to compute the divergence of the enthalpy flux which for sufficent low velocity gives the convective contribution to the energy balance. Since the radiative losses are known as a function of pressurc and temperature, the balance among all these terms and the enegy supply along the loop allows the determination of this latter, if the quasi stationary hypotesis is valid. Fig. 3 shows the trend of the energy' balance terms (ergs $\mathrm{cm}^{-3} \mathrm{sec}^{-1}$ ) along the loop as a function of temperature at time $00^{\mathrm{h}} 06^{\mathrm{m}}$; the full line indicates the 
radiative losses as computed using Landini and Monsignori Fossi spectral code (1984); the dotted line gives the divergence of the conductive flux which may be deduced from the model; dashed and dolled line gives the divergence of the enthalpy flux $E_{e n t}=\rho_{0} v_{0} R \gamma /(\gamma-1) d T / d l$, where $v_{0}$. is the velocity deduced from CIV dopplergrans and $\rho_{O}$ the corresponding density; dashed line is the power supply deduced from the balance.

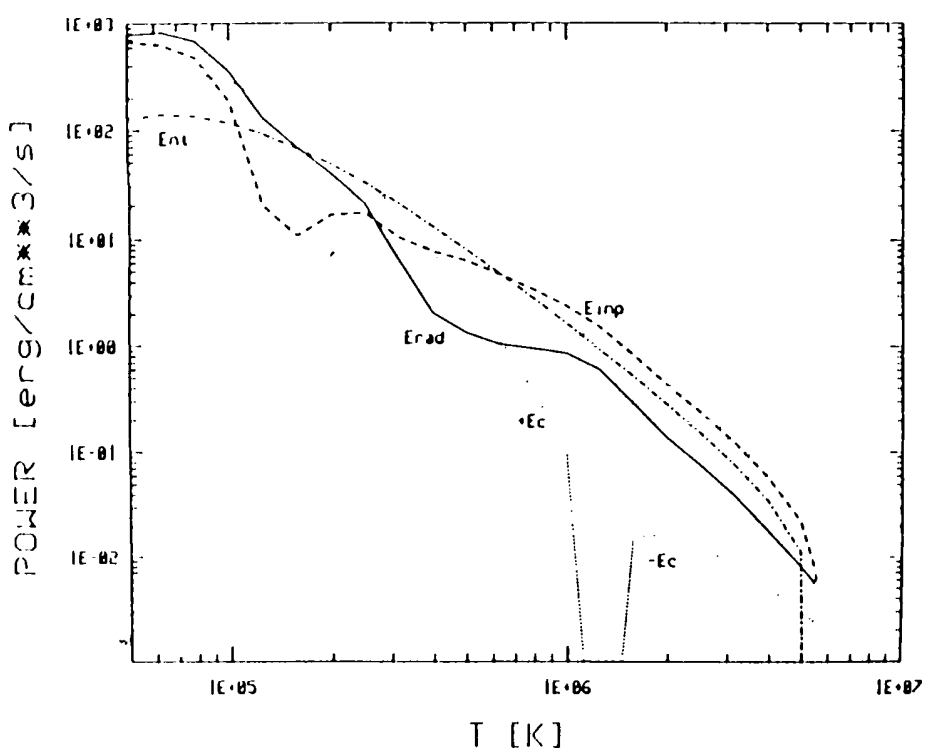

fig. 3

Clear indication exists that a power supply is necessary over all the coronal part of the loop $\left(\mathrm{T}_{\mathrm{C}}>10^{6} \mathrm{~K}\right.$ ) where it depends on the temperature with a power law very near to $\mathrm{T}^{-2.5}$ (or $\mathrm{n}^{2.5}$ due to the costam pressure condition)

\section{CONCLUSIONS}

Pressure of the order of $10 \mathrm{dy}^{\prime} \mathrm{cm}^{-2}$ and maximum temperature around $510^{6} \mathrm{O}_{\mathrm{K}}$ are obtained in this low cvolving fare; both temperature and pressure increalse during the cvent: almost twince the pressure and less than $30 \%$ the temperature indicating a gradual increase of density (evaporation from the lecl???)

Since the time evolution of the temperature is very slow a stationary analysis is performed, the conductive, convective and radiative terms of the energy balance are evaluated and the power supply along the loop is estimated. Over most of the coronal region of the loop a continuos power supply is needed which is not costant and follows approximatly a power low of the temperature about $\mathrm{T}^{-2.5}$ ( or $n^{2.5}$ since oncesure is cos(ant).

\section{REFERENCES}

Landini M. and Monsignori Fossi B.C., 1984, Physica Scripta T7,53.

Landini M., Monsignori Fossi B.C., Paresce F. and Stern R.:1985,Ap.J.289,709

Monsignori Fossi B.C. and Landini M.:1988 in "Activity in cool star envelopes" O.Havens et al. eds.,237

Rosner R.,Tucker W.II. and Vaiana G.S.,1978, Ap.J., 220,643 